\title{
Analyticity of solutions for quasilinear wave equations and other quasilinear systems
}

\author{
Sergei Kuksin, Nikolai Nadirashvili ${ }^{\dagger}$
}

September 4, 2018

\begin{abstract}
We prove the persistence of analyticity for classical solution of the Cauchy problem for quasilinear wave equations with analytic data. Our results show that the analyticity of solutions, stated by the Cauchy-Kowalewski and Ovsiannikov-Nirenberg theorems, lasts till a classical solution exists. Moreover, they show that if the equation and the Cauchy data are analytic only in a part of space-variables, then a classical solution also is analytic in these variables. The approach applies to other quasilinear equations and implies the persistence of the space-analyticity (and the partial space-analyticity) of their classical solutions.
\end{abstract}

\section{Introduction}

Consider a quasilinear wave equation:

$$
\begin{gathered}
\square u+f(t, x, u, \nabla u, \dot{u})=0, \quad \operatorname{dim} x=d, \quad t \in \mathbb{R}, \\
u_{t=0}=u_{0}, \quad \dot{u}_{t=0}=u_{1},
\end{gathered}
$$

where $f$ is a real-analytic function of all its arguments and the Cauchy data $u_{0}, u_{1}$ are realanalytic functions of $x$. To begin with we assume the periodic boundary conditions:

$$
x \in \mathbb{T}^{d}=\mathbb{T}_{\Gamma}^{d}=\mathbb{R}^{d} / \Gamma \quad(\Gamma \text { is a lattice }) .
$$

Regarding the solvability of the Cauchy problem (0.1), (0.2) two facts are well known: the life-span of its classical solution is non zero, i.e., there is $T>0$ such that on $[0, T)$ the problem (0.1), (0.2) has a $C^{2}$-solution $u$, e.g. see [Hör97 and Proposition 1.1 below. On the other hand by the Cauchy-Kowalewski theorem [Kow75] there is a positive $\varepsilon_{1}$ such that for $t \in\left[0, \varepsilon_{1}\right)$ the solution $u$ is real-analytic. Ovsiannikov and Nirenberg gave a beautiful generalization of the latter theorem to equations (0.1), where the nonlinearity $f$ is continuous in $t$ (and still is

\footnotetext{
*Université Paris 7 UFR de Mathmatiques, 75205 Paris Cedex 13, France, e-mail: kuksin@gmail.com
}

${ }^{\dagger}$ LATP, CMI, 39, rue F. Joliot-Curie, 13453 Marseille France, e-mail: nicolas@cmi.univ-mrs.fr 
analytic in other variables), see in [Nis77. By their result, for any real-analytic $u_{0}$ and $u_{1}$ there is a positive $\varepsilon_{2}$ such that for $t \in\left[0, \varepsilon_{2}\right)$ the solution $u$ is real-analytic in $x$.

From the proofs of the Cauchy-Kowalewski and Ovsiannikov-Nireberg theorems all that one can say about the life-spans of analyticity $\varepsilon_{1}$ and $\varepsilon_{2}$ is just their positivity. However, for the different classes of quasilinear wave equations, supplemented with sufficiently smooth initial data, the life-span $T$ of classical (smooth) solutions is often fairly large, sometimes $T=\infty$. The natural question is if the range of analyticity is extendable up to $T$.

By a general result of Alinhac and Metivier AM84 the life-span of analyticity $\varepsilon_{1}$ in the Cauchy-Kowalewski theorem is equal to $T$. The proof of their theorem is very technical and involves complicated recombination of the Taylor's coefficients. (In BB77] a similar result was obtained earlier for solutions of the $2 \mathrm{~d}$ Euler equation, using hyperbolic features of that equation.) The aim of this paper is to give a short and transparent proof of this (and actually more general) properties of solutions of quasilinear wave equations. We also show that the life-span of analyticity $\varepsilon_{2}$ in the Ovsiannikov-Nirenberg theorem equals the life-span time $T$.

Let $f$ be continuous in $t, H^{m}$-smooth in $x$ and analytic in $u, \nabla u, \dot{u}$ (see Section 1.1). Let $u(t, x), 0 \leq t \leq T, x \in \mathbb{T}^{d}$, be a solution of the Cauchy problem (0.1), (0.2) which defines a continuous curve $t \mapsto u(t, \cdot) \in H^{m+1}\left(\mathbb{T}^{d}\right), \quad C^{1}$-smooth as a curve in $H^{m}\left(\mathbb{T}^{d}\right)$. Assume that $m>d / 2$ (so $u(t, x)$ is a continuos function, $C^{1}$-smooth in $\left.t\right)$.

Theorem 0.1. i) If $f$ and $u_{0}, u_{1}$ are real-analytic in $\left(x_{1}, \ldots, x_{k}\right), 1 \leq k \leq d$, then $u$ also is real-analytic in these variables,

ii) if $f$ and $u_{0}, u_{1}$ are real-analytic in all their arguments, then $u$ also is.

Note that the first assertion of the theorem and the local in time existence of a classical solution imply that if $u_{0}, u_{1}$ and $f$ are sufficiently smooth in $x$, continuous in $t$ and analytic in $x_{1}, \ldots, x_{k}, u, \nabla u$ and $\dot{u}$, then the problem (0.1), (0.2) has a unique local in time solution, analytic in $x_{1}, \ldots, x_{k}$ (see below Corollary 1.9). This generalises the Ovsiannikov-Nirenberg theorem for equations of such class.

Theorem 0.1 is proved in Section 1; its proof is based on properties of the nonlinear semigroup, generated by the problem (0.1), (0.2). In Theorem 2.2. Section 2, we show that the assertion holds for solutions of (0.1), (0.2) defined locally, in a characteristic cone in $\mathbb{R} \times \mathbb{R}^{d}$. The local result on the analyticity implies the analyticity of global solutions defined on the whole torus. It straightforwardly generalizes to equations on homogeneous spaces and implies the corresponding global results. For example, Theorem 0.1,ii) remain true for equations in the standard sphere $S^{d}$, see Section 3.1

We preface Theorem 0.1 to local Theorem 2.2 since the assertion i) of the former and its proof remain true for other classes of equations for which the latter is no more valid. E.g. see Section 3 for quasilinear parabolic equations, the 3d Navier-Stokes system and NLS equations. In the same time, the proof of assertion ii) does not generalise to non-hyperbolic equations (and for quasilinear parabolic equations its claim is wrong). So while the Cauchy-Kowalewski theorem is an assertion about hyperbolic equations, the Ovsiannikov-Nirenberg theorem describes a general property of a large class of quasilinear systems.

We note that similar $C^{\infty}$-smooth properties of solutions for (0.1), (0.2) are known, see Koc93, Sog08.

Our proof relies heavily on the theory of analytic mappings in Banach spaces. For the reader's convenience we summarised it in an appendix to this paper. 
Acknowledgments. The authors would like to thank H. Koch for very useful discussions. SK was supported l'Agence Nationale de la Recherche through the grant ANR-10-BLAN 0102.

\section{Global results: quasilinear wave equation on $\mathbb{T}^{d}$}

\subsection{Single equation.}

Here we study the Cauchy problem for a quasilinear wave equation (0.1), (0.2) on $\mathbb{T}^{d}=\mathbb{R}^{d} / \Gamma$, where the function $f$ is continuous in all variables, is $H^{m}$ smooth in $x$, where

$$
m>d / 2 \quad(\text { or } m>d / 2-1 \text { if } f \text { does not depend on } \nabla u \text { and } \dot{u}),
$$

and is (real-)analytic in the arguments $u, \nabla u, \dot{u}$. 1 We denote by $H^{m}$ the Sobolev spaces $H^{m}\left(\mathbb{T}^{d}\right)$ with the norm $\|u\|_{m}=\left(\left|\nabla^{m} u\right|_{L_{2}}^{2}+|u|_{L_{2}}^{2}\right)^{1 / 2}$, and abbreviate $H^{m+1} \times H^{m}=\mathcal{H}^{m}$. Consider the Cauchy operator for the linear wave equation:

$$
\widetilde{\square}: u \mapsto\left(u_{t=0}, \dot{u}_{t=0}, \square u\right) .
$$

It is well known that for any reasonable domain of definition this map is an embedding. For any $T>0$ consider the spaces

$$
X_{m}^{T}=C\left(0, T ; H^{m+1}\right) \cap C^{1}\left(0, T ; H^{m}\right), \quad Y_{m}^{T}=\mathcal{H}^{m} \times C\left(0, T ; H^{m}\right) .
$$

It is also well known (e.g., see [Tem97]) that the inverse operator defines a continuous mapping

$$
\tilde{\square}^{-1}: Y_{m}^{T} \rightarrow X_{m}^{T}
$$

(but certainly $\widetilde{\square}$ does not map $X_{m}^{T}$ to $Y_{m}^{T}$ ).

The spaces $Y_{m}^{T}$ and $X_{m}^{T}$ suit well to study solvability of the problem (0.1), (0.2). Indeed, denote $\varkappa=\left|\left(u_{0}, u_{1}\right)\right|_{\mathcal{H}^{m}}$, assume that $u_{0}, u_{1}$ are smooth and that $u(t, x)$ is a smooth solution of the problem such that

$$
|U(t)|_{\mathcal{H}^{m}} \leq 2 \varkappa \quad \forall 0 \leq t \leq T^{\prime}
$$

with some $T^{\prime}>0$, where $U(t)=(u(t), \dot{u}(t))$. Taking the $H^{m}$ scalar-product of (0.1) with $\dot{u}(t)$, we get that

$$
\frac{1}{2} \frac{d}{d t}\|\dot{u}\|_{m}^{2}+C \frac{d}{d t}\|u\|_{m+1}^{2} \leq C_{1}\|u\|_{m}\|\dot{u}\|_{m}+C_{2}\|f(t, x, u, \nabla u, \dot{u})\|_{m}\|\dot{u}\|_{m},
$$

for suitable contants $C, C_{1}, C_{2}$. By the apriori assumption and since the space $H^{m}$ is an algebra, for $0 \leq t \leq T^{\prime}$ the r.h.s. is bounded by $C(\varkappa)$. Therefore $|U(t)|_{\mathcal{H}^{m}}^{2} \leq \varkappa^{2}+t C_{1}(\varkappa)$. So there exists $T(\varkappa)>0$ such that $|U(t)|_{\mathcal{H}^{m}} \leq 2 \varkappa$ for $0 \leq t \leq T(\varkappa)$. In the usual way the obtained apriori estimate implies

Proposition 1.1. There exists $T^{\prime}>0$, depending only on $f$ and $\left|\left(u_{0}, u_{1}\right)\right|_{\mathcal{H}^{m}}$, such that the problem (0.1), (0.2) has a unique solution $u(t, x), 0 \leq t \leq T^{\prime}$, belonging to the space $Y_{m}^{T^{\prime}}$.

\footnotetext{
${ }^{1}$ More precisely, the function $f(t, x, u, \xi, \eta)$ is continuous and analytically in $\mathfrak{z}=(u, \xi, \eta)$ extends to a neighbourhood $Q$ of $\mathbb{R}^{d+2}$ in $\mathbb{C}^{d+2}$. The extended function is bounded uniformly on sets $[0, T] \times \mathbb{T}^{d} \times Q_{R}$, where $Q_{R}=Q \cap\left\{\mathfrak{z} \in \mathbb{C}^{d+2}:|\mathfrak{z}|<R\right\}$. Moreover, $\|f(t, \cdot, \mathfrak{z})\|_{m} \leq C(R)$ for all $t \in[0, T]$ and $\mathfrak{z} \in Q_{R}$, for each $R>0$.
} 
It is well known that in general the local solution $u(t)$ cannot be extended to all $t \geq 0$. The construction below gives a convenient implicit description of the set of initial data for which a solution exists for $0 \leq t \leq T$. This construction is a part of the $\mathrm{PhD}$ thesis of the first author Kuk81.

Denote $\widetilde{\square}^{-1} Y_{m}^{T}=Z_{m}^{T}$ and provide $Z_{m}^{T}$ with a norm, induces from $Y_{m}^{T}$ by $\widetilde{\square}^{-1}$. This is a Banach space,

$$
\begin{gathered}
\widetilde{\square}: Z_{m}^{T} \rightarrow Y_{m}^{T} \quad \text { is an isomorphism, } \\
Z_{m}^{T} \subset X_{m}^{T} \quad \text { continuously }
\end{gathered}
$$

by (1.2), and $X_{m+1}^{T} \subset Z_{m}^{T}$. Denote by $F$ the nonlinear differential operator $F(u)=f(t, x, u, \nabla u, \dot{u})$ and by $\Phi-$ the operator of the Cauchy problem (0.1), (0.2). That is

$$
\Phi(u)=\widetilde{\square}(u)+(0,0, F(u)) .
$$

Since $m>d / 2$, then the space $C\left(0, T ; H^{m}\right)$ is a Banach algebra. Using (1.3) and (1.4) we get that the mapping

$$
\Phi: Z_{m}^{T} \rightarrow Y_{m}^{T} \quad \text { is analytic }
$$

(e.g. see RS96; cf. Exemple 4.3 in Appendix). It is well known that the Cauchy problem (0.1), (0.2) with zero in the r.h.s. replaced by any function from $C\left(0, T ; H^{m}\right)$ has at most one solution in $X_{m}^{T}$. So $\Phi$ is an embedding. Consider its differential in any point $u \in Z_{m}^{T}$ :

$$
d \Phi(u)(v)=\left(v_{t=0}, \dot{v}_{t=0}, \square v+d_{3} f[u] v+d_{4} f[u] \nabla v+d_{5} f[u] \dot{v}\right) .
$$

Here $f[u]=f(x, u, \nabla u, \dot{u})$ and $d_{j}$ denotes the differential with respect to the $j$-th variable.

Lemma 1.2. For any $u \in Z_{m}^{T}$ the $\operatorname{map} d \Phi(u): Z_{m}^{T} \rightarrow Y_{m}^{T}$ is an isomorphism.

Proof. For any $\left(v_{0}, v_{1}, g\right) \in Y_{m}^{T}$ consider the corresponding Cauchy problem which we write as

$$
\square v+v+\left(d_{3} f[u] v+d_{4} f[u] \nabla v+d_{5} f[u] \dot{v}-v\right)=g, \quad v(0)=v_{0}, \dot{v}(0)=v_{1} .
$$

Let $v_{0}, v_{1}$ and $g$ be smooth and $v$ be a smooth solution of the problem. Multiplying the equation by $\dot{v}$ in $H^{m}$ and using that the space $H^{m}$ is an algebra, we get:

$$
\frac{1}{2} \frac{d}{d t}\|\dot{v}\|_{m}^{2}+C \frac{d}{d t}\|v\|_{m+1}^{2} \leq C_{2}\|v\|_{m}^{2}+C_{3}\|v\|_{m+1}\|v\|_{m}+C_{4}\|\dot{v}\|_{m}^{2}+\|g\|_{m}^{2},
$$

where the constants $C_{j}$ are continuous functions of $\|u\|_{X_{m}^{T}}$. We immediately get from this relation that

$$
\|v\|_{X_{m}^{T}} \leq C\left(\|u\|_{X_{m}^{T}}\right)\left\|\left(v_{0}, v_{1}, g\right)\right\|_{Y_{m}^{T}}
$$

In the usual way this apriori estimate implies that (1.8) has a unique solution $v \in X_{m}^{T}$. Then we see from (1.8) that $\widetilde{\square} v \in Y_{m}^{T}$. So $v \in Z_{m}^{T}$ and $\|v\|_{Z_{m}^{T}} \leq C^{\prime}\left(\|u\|_{Z_{m}^{T}}\right)\left\|\left(v_{0}, v_{1}, g\right)\right\|_{Y_{m}^{T}}$.

Since $\Phi$ is an embedding, then Lemma 1.2 jointly with the inverse function theorem (see Theorem 4.5) imply

Lemma 1.3. The mapping $\Phi$ is an analytic diffeomorphism of the space $Z_{m}^{T}$ and a domain $\mathcal{O} \in Y_{m}^{T}$. 
Therefore if for some $\left(u_{1}, u_{2}, g\right) \in Y_{m}^{T}$ the problem (0.1), (0.2) with zero in the r.h.s. of (0.1) replaced by $g$ has a solution $u \in X_{m}^{T}$, then $\left(u_{1}, u_{2}, g\right) \in \mathcal{O}, u$ belongs to $Z_{m}^{T}$ and analytically depends on $\left(u_{1}, u_{2}, g\right)$. Denote

$$
\mathcal{O}^{0}=\left\{\left(u_{0}, u_{1}\right) \in \mathcal{H}^{m}:\left(u_{0}, u_{1}, 0\right) \in \mathcal{O}\right\} .
$$

Then for $0 \leq t \leq T$ the flow-maps

$$
S_{0}^{t}: \mathcal{O}^{0} \rightarrow \mathcal{H}^{m}, \quad\left(u_{0}, u_{1}\right) \rightarrow(u(t), \dot{u}(t)),
$$

are well defined and analytic.

We recall that $T$ is any positive number and that the domain $\mathcal{O}^{0}$ depends on the timeinterval $[0, T], \mathcal{O}^{0}=\mathcal{O}^{0}([0, T])$. Similar we may study solutions of (0.1), (0.2) on negative time-intervals $[-T, 0]$. The assertions above remain true for operators $S_{0}^{t}$ with $t \in[-T, 0]$ and with the domain $\mathcal{O}^{0}=\mathcal{O}^{0}([-T, 0])$. Finally, we may consider eq. (0.1) with the Cauchy data given not at $t=0$, but at $t=t_{1}$, for arbitrary $t_{1} \in[0, T]$. In this way we find that the flow-maps $S_{t_{1}}^{t_{2}}$, where $t_{1}, t_{2} \in[0, T]$, are analytic operators with domains $\mathcal{O}^{t_{1}}([0, T])$.

It is clear from our construction that the operators $S_{t_{1}}^{t_{2}}$ and $S_{t_{2}}^{t_{1}}$ with domains $\mathcal{O}^{t_{1}}([0, T])$ and $\mathcal{O}^{t_{2}}([0, T])$ are inverse to each other, and that an operator $S_{t_{1}}^{t_{2}}$ analytically extends to the bigger domain $\mathcal{O}^{t_{1}}\left(\left[t_{1}, t_{2}\right]\right)$. This is its maximal domain of definition.

\subsection{Families of equations.}

We fix any $k \in\{1, \ldots, d\}$ and assume that

$$
\mathbb{T}^{d}=\mathbb{T}^{k} \times \mathbb{T}^{d-k}
$$

(that is, $\Gamma=\Gamma_{k} \oplus \Gamma_{d-k}$ and $\mathbb{T}^{k}=\mathbb{R}^{k} / \Gamma_{k}, \mathbb{T}^{d-k}=\mathbb{R}^{d-k} / \Gamma_{d-k}$ ). We make the torus $\mathbb{T}^{k}=\{\theta=$ $\left.\left(\theta_{1}, \ldots, \theta_{k}\right)\right\}$ to act on $\mathbb{T}^{d}$ by the shifts ${ }_{\theta} R$,

$$
{ }_{\theta} R(x)=\left(x^{I}+\theta, x^{I I}\right),
$$

where $x^{I}=\left(x_{1}, \ldots, x_{k}\right)$ and $x^{I I}=\left(x_{k+1}, \ldots, x_{d}\right)$. Then the torus acts on the operators $F$ by shifting their coefficients: $\left({ }_{\theta} R F\right)(u)=f\left(t,{ }_{\theta} R x, u, \nabla u, \dot{u}\right)$. Clearly we have

$$
\left(\square+{ }_{\theta} R F\right)\left({ }_{\theta} R u\right)={ }_{\theta} R((\square+F)(u)) .
$$

The operators of the shifted Cauchy problem ${ }_{\theta} \Phi(u)=\widetilde{\square} u+\left(0,0,\left({ }_{\theta} R F\right) u\right)$ define a mapping

$$
\bar{\Phi}^{1}: \mathbb{T}^{k} \times Z_{m}^{T} \rightarrow Y_{m}^{T}, \quad(\theta, u) \rightarrow{ }_{\theta} \Phi(u) .
$$

Lemma 1.4. Assume that the function $f(t, x, u, \nabla u, \dot{u})$ is analytic in $x^{I}$. Then the mapping $\bar{\Phi}^{1}$ is analytic.

Proof. By (1.6) and Corollary 4.4 we only have to check that the mapping is locally bounded and is analytic in $\theta$. The local boundedness of this map (and its continuity) follow from the Banach algebra property of the spaces $H^{s}$ with $s>d / 2$, e.g. see [RS96; cf. (4.2). Since $f$ is analytic in $x^{I}$, then ${ }_{\theta} \Phi(u)$ complex-analytically depends on $\theta$ from the complex vicinity of $\mathbb{T}^{k}$. This implies the assertion. 
By the results of Section 1.2, for any $\theta$ the operator $\bar{\Phi}^{1}(\theta, \cdot)$ defines an analytic diffeomorphism of $Z_{m}^{T}$ and a domain ${ }_{\theta} \mathcal{O} \subset Y_{m}^{T}$. By the implicit function theorem

$$
\text { the mapping } \quad{ }_{\theta} \mathcal{O} \ni \xi \rightarrow\left(\bar{\Phi}^{1}(\theta, \cdot)\right)^{-1} \in Z_{m}^{T} \quad \text { is analytic in } \xi \text { and } \theta .
$$

Denoting ${ }_{\theta} \mathcal{O}^{0}=\left\{\left(u_{0}, u_{1}\right):\left(u_{0}, u_{1}, 0\right) \in{ }_{\theta} \mathcal{O}\right\}$ we see that for $\theta \in \mathbb{T}^{k}$ and $0 \leq t \leq T$ the time- $t$ flow-mapping, corresponding to the nonlinearity ${ }_{\theta} R F$, is

an analytic transformation ${ }_{\theta} S_{0}^{t}:{ }_{\theta} \mathcal{O}^{0} \rightarrow \mathcal{H}^{m}$ which analytically depends on $\theta$.

Relation (1.10), where $(\square+F) u=0$, implies that

$$
{ }_{\theta} S_{0}^{t} \circ{ }_{\theta} R={ }_{\theta} R \circ S_{0}^{t} .
$$

In particular, ${ }_{\theta} R \mathcal{O}^{0}={ }_{\theta} \mathcal{O}^{0}$.

Similar for any $\delta \in \mathbb{R}$ we define

$$
\left({ }^{\delta} R F\right)(u)=f(t+\delta, x, u, \nabla u, \dot{u}), \quad{ }^{\delta} \Phi=\widetilde{\square}+\left(0,0,{ }^{\delta} R F\right) .
$$

Assume that there exists $\rho>0$ such that for each value of $(x, u, \nabla u, \dot{u})$ the function $t \mapsto$ $f(t, x, u, \nabla u, \dot{u})$ analytically extends to the segment $[-\rho, T+\rho]$. Then the mapping

$$
\bar{\Phi}^{2}:(-\rho, \rho) \times Z_{m}^{T} \rightarrow Y_{m}^{T}, \quad(\delta, u) \mapsto{ }^{\delta} \Phi(u),
$$

is analytic, and for any $|\delta|<\rho$ it defines an analytic isomorphism

$$
{ }^{\delta} \Phi: Z_{m}^{T} \rightarrow{ }^{\delta} \mathcal{O} \subset Y_{m}^{T},
$$

which analytically depends on $\delta$. We set ${ }^{\delta} \mathcal{O}^{0}=\left\{\left(u_{0}, u_{1}\right) \in \mathcal{H}^{m}:\left(u_{0}, u_{1}, 0\right) \in{ }^{\delta} \mathcal{O}\right\}$ and denote by ${ }^{\delta} S_{0}^{t}$ the mapping $S_{0}^{t}$, corresponding to the operator ${ }^{\delta} R F$. Then

the operator ${ }^{\delta} S_{0}^{t}:{ }^{\delta} \mathcal{O}^{0} \rightarrow \mathcal{H}^{m}$ is analytic and analytically depends on $\delta \in(-\rho, \rho)$.

\subsection{Analyticity of solutions}

There is a delicate difference between the smoothness (or analyticity) of solutions for a nonlinear wave equation in time and in space. For instance, there is a number of results which imply for a solution a high smoothness in $x$ and only a limited smoothness in $t$, see Hör97. Another example is given by the Ovsiannikov-Nirenberg theorem. Accordingly below we consider smoothness of solutions for (0.1), (0.2) in $x$ and in $t$ separately.

Space-analyticity. Assume that the function $f$ as above is analytic in $x^{I}$, as well as the initial data $u_{0}$ and $u_{1}$. Assume also that the problem (0.1), (0.2) has a solution $u \in Y_{m}^{T}$. Then $\left(u_{0}, u_{1}\right) \in{ }_{\theta} \mathcal{O}^{0}$ and by (1.13) ${ }_{\theta} S_{0}^{t} u_{0}$ with $0 \leq t \leq T$ is well defined for $\theta$ from a small ball $B_{\varepsilon}=\{|\theta|<\varepsilon\}$ and is analytic in $\theta$. Using (1.14) we have

$$
u\left(t, x^{I}+\theta, x^{I I}\right)=\left({ }_{\theta} R \circ S_{0}^{t}\right)\left(u_{0}, u_{1}\right)(x)=\left({ }_{\theta} S_{0}^{t} \circ{ }_{\theta} R\right)\left(u_{0}, u_{1}\right)(x) .
$$

Since $u_{0}$ is analytic in $x^{I}$, then the mapping $\mathbb{T}^{k} \rightarrow \mathcal{H}^{m}, \quad B_{\varepsilon} \ni \theta \mapsto{ }_{\theta} R\left(u_{0}, u_{1}\right)$, is analytic. Using (1.13) we get 
Theorem 1.5. Assume that the nonlinearity $f$ and the initial data $u_{0}, u_{1}$ are analytic in $x^{I} \in$ $\mathbb{T}^{k}$ and the problem (0.1), (0.2) has a solution $u(t, x) \in Y_{m}^{T}, 0 \leq t \leq T, x \in \mathbb{T}^{d}$. Then $u$ is analytic in $x^{I}$.

Time-analyticity. Now assume that for a suitable $\rho>0$ the function $f(t, x, u, \nabla u, \dot{u})$, where $-\rho \leq t \leq T+\rho$, is analytic in all its arguments, that the Cauchy data $u_{0}(x)$ and $u_{1}(x)$ are analytic and that the problem (0.1), (0.2) has a solution $u \in Y_{m}^{T}$. Denote $U(t)=(u(t), \dot{u}(t))$. By the Cauchy-Kowalewski theorem, the function $u(t, x)$ is analytic for $|t|<\varepsilon$ and $x \in \mathbb{T}^{d}$ with a suitable $\varepsilon>0$. Therefore the curve $[0, T] \rightarrow \mathcal{H}^{m}, t \mapsto U(t)$, also is analytic for $|t|<\varepsilon$. For any $t_{*} \in[0, T]$ we write the solution $U(t)$ for $t$ close to $t_{*}$ as $U\left(t_{*}+\tau\right)={ }^{\tau} S_{0}^{t_{*}} \circ U(\tau)$. Using (1.15) we get

Lemma 1.6. Under the above assumptions the curve $[0, T] \rightarrow \mathcal{H}^{m}, t \rightarrow(u, \dot{u})(t)$, is analytic. In particular, $u(t, x)$ is analytic in $t \in[0, T]$ for each $x$.

This result and Theorem 1.5 imply

Theorem 1.7. Assume that the nonlinearity $f(t, x, u, \nabla u, \dot{u})$, where $-\rho \leq t \leq T+\rho$, and the initial data $u_{0}, u_{1}$ are analytic in all variables and the problem (0.1), (0.2) has a solution $u(t, x) \in Y_{m}^{T}$. Then $u(t, x)$ is an analytic function in all its arguments.

We recall that for any $t_{1}, t_{2} \in[0, T]$ the flow-map $S_{t_{1}}^{t_{2}}$ defines an analytical isomorphism $\mathcal{O}^{t_{1}}([0, T]) \rightleftharpoons \mathcal{O}^{t_{2}}([0, T])$. Denote by $\mathcal{A}\left(\mathbb{T}^{d}\right)$ the space of analytic functions on $\mathbb{T}^{d}$.

Corollary 1.8. If the nonlinearity $f$ is analytic in $x, u, \nabla u, \dot{u}$, then the mapping $S_{t_{1}}^{t_{2}}$ defines a bijection $\mathcal{O}^{t_{1}}([0, T]) \cap \mathcal{A}\left(\mathbb{T}^{d}\right) \vec{\sim} \mathcal{O}^{t_{2}}([0, T]) \cap \mathcal{A}\left(\mathbb{T}^{d}\right)$. If $f$ is also analytic in $t \in[-\rho, T+\rho]$, then for each $u \in \mathcal{O}^{t_{1}}([0, T]) \cap \mathcal{A}\left(\mathbb{T}^{d}\right)$ the curve $\left(t_{1}, t_{2}\right) \mapsto S_{t_{1}}^{t_{2}}(u)$ is analytic in $t_{1}$ and $t_{2}$.

Combining Theorem [1.5 with Proposition 1.1 we get

Corollary 1.9. Let the nonlinearity $f$ and the Cauchy data $u_{0}, u_{1}$ be as in Theorem 1.5. Then there exists $T^{\prime}>0$ such that for $0 \leq t \leq T^{\prime}$ the Cauchy problem has a unique solution $u \in Y_{m}^{T^{\prime}}$, which is analytic in $x^{I}$.

The global results above generalise to other classes of quasilinear PDE. E.g., to quasilinear parabolic and Schrödinger equations, see Section 3 ,

\section{Local results}

\subsection{Equations in characteristic cones.}

In this section we consider the problem (0.1), (0.2) defined in a characteristic cone in $\mathbb{R}^{d+1}$.

Let $T>0$ and $0<a<T$. Denote by $K$ a truncated characteristic cone:

$$
K=K(T, a)=\left\{(t, x) \in[0, T-a] \times \mathbb{R}^{d}:|x| \leq T-t\right\}
$$

(below for short we call it a cone). Denote by $B_{r} \subset \mathbb{R}^{d}$ the closed ball of radius $r$ centered in the origin and denote $b^{t}=B_{T-t}$. In this section we study the problem (0.1), (0.2) in the cone $K$, where the Cauchy data are given on the ball $K \cap\{t=0\}$, identified with $b^{0}$. The nonlinearity 
$f$ is assumed to be analytic in all its variables and analytically extendable to $U_{\varepsilon}(K) \times \mathbb{R}^{d+2}$, where $U_{\varepsilon}(K)$ is the $\varepsilon$-vicinity of $K$ in $\mathbb{R}^{d+1}, \varepsilon>0$. Then for a given Cauchy data the problem (0.1), (0.2) has at most one classical solution, [Hör97. Our goal is to prove for this solution Theorem 0.1, assuming that the Cauchy data also are analytic.

Denote by $\operatorname{Tr}_{\rho}(g)$ the restriction of a function $g(x)$ to the ball $B_{\rho}, \rho>0$. It is well known that for $k \geq 0$ there exists a bounded linear map

$$
L_{1}^{k}: H^{k}\left(B_{1}\right) \rightarrow H_{0}^{k}\left(B_{2}\right)
$$

such that $\operatorname{Tr}_{1} \circ L_{1}^{k}=$ id and $L_{1}^{k} u(x)=0$ for $|x| \geq \frac{3}{2}$. For $0<\rho \leq T$ we denote by $L_{\rho}^{k}$ the linear operator $L_{\rho}^{k}: H^{k}\left(B_{\rho}\right) \rightarrow H_{0}^{k}\left(B_{2 \rho}\right) \subset H_{0}^{k}\left(B_{2 T}\right)$, obtained from $L_{1}^{k}$ by the dilation (so $\left.\operatorname{Tr}_{\rho} \circ L_{\rho}^{k}=\mathrm{id}\right)$. Denote by $C\left([0, T-a] ; H^{k}\left(b^{t}\right)\right)$ the space of functions $u(t, x)$ on the cone $K$, satisfying 2

$$
\mathcal{L}^{k} u \in C\left([0, T-a] ; H_{0}^{k}\left(B_{2 T}\right)\right) \quad \text { where } \quad\left(\mathcal{L}^{k} u\right)(t)=L_{T-t}^{k}(u(t)),
$$

and provide this space with the norm, induced from $C\left([0, T-a] ; H^{k}\left(B_{2 T}\right)\right)$. Next, for $m>d / 2$ denote $\mathcal{H}^{m}=H^{m+1}\left(b^{0}\right) \times H^{m}\left(b^{0}\right)$ and set

$$
\begin{aligned}
& X_{m}^{\prime}=\left\{u \in C\left([0, T-a] ; H^{m+1}\left(b^{t}\right)\right): \dot{u} \in C\left([0, T-a] ; H^{m}\left(b^{t}\right)\right)\right\}, \\
& Y_{m}^{\prime}=\mathcal{H}^{m} \times C\left([0, T-a] ; H^{m}\left(b^{t}\right)\right) .
\end{aligned}
$$

We denote by $\widetilde{\square}^{\prime}$ the operator of the Cauchy problem for $\square$ in $K$ which sends any function $u(t, x) \in X_{m}^{\prime}$ to $\widetilde{\square}^{\prime} u=(u(0), \dot{u}(0), \square u) \in Y_{m-1}^{\prime}$.

Consider the torus $T^{d}=\mathbb{R}^{d} /(4 T) \mathbb{Z}^{d}$, the corresponding spaces $X_{m}^{T}, Y_{m}^{T}, Z_{m}^{T}$ and the operator $\widetilde{\square}$. We will identify functions on $T^{d}$ with $4 T$-periodic functions on $\mathbb{R}^{d}$. Denoting by $\operatorname{Tr}$ the operator of restricting a function on $[0, T-a] \times \mathbb{R}^{d}$ to $K$, we get the mappings

$$
\operatorname{Tr}: X_{m}^{T} \rightarrow X_{m}^{\prime}, \quad \operatorname{Tr}: Y_{m}^{T} \rightarrow Y_{m}^{\prime} .
$$

For $\rho \leq T$ and any $u(x) \in H_{0}^{s}\left(B_{2 \rho}\right)$ denote by $\iota$ the operator which first extends $u(x)$ to the cube $[-2 T, 2 T]^{d}$ by zero outside the ball $B_{2 \rho}$ and next extends it to a $4 T$-periodic function. This is a bounded linear operator from $H_{0}^{s}\left(B_{2 \rho}\right)$ to $H^{s}\left(T^{d}\right)$ for any $s \geq 0$ and any $0<\rho \leq T$. For a function $u(t, x)$ we set $(\iota u)(t, x)=\iota(u(t, \cdot))(x)$, if the r.h.s. is defined. Clearly $\operatorname{Tr} \circ \iota=\mathrm{id}$ on the space $C\left([0, T-a] ; H^{m}\left(b^{t}\right)\right)$.

Lemma 2.1. The inverse operator $\left(\widetilde{\square}^{\prime}\right)^{-1}$ equals

$$
\left(\widetilde{\square}^{\prime}\right)^{-1}=\operatorname{Tr} \circ \widetilde{\square}^{-1} \circ \iota .
$$

It defines a continuous mapping $\left(\widetilde{\square}^{\prime}\right)^{-1}: Y_{m}^{\prime} \rightarrow X_{m}^{\prime}$.

Proof. For $\left(u_{0}, u_{1}, g\right) \in Y_{m}^{\prime}$ consider functions $\hat{u}_{j}(x)=\iota\left(L_{T}^{m}\left(u_{j}(x)\right)\right), j=0,1$ and $\hat{g}(t, x)=$ $\iota\left(\mathcal{L}^{m}(g)\right)$. Then $\left|\left(\hat{u}_{0}, \hat{u}_{1}, \hat{g}\right)\right|_{Y_{m}^{T}} \leq C\left|\left(u_{0}, u_{1}, g\right)\right|_{Y_{m}^{\prime}}$. The solution of the Cauchy problem for $\square$ in $T^{d}, U(t, x)=\widetilde{\square}^{-1}\left(\hat{u}_{0}, \hat{u}_{1}, \hat{g}\right)$, satisfies $|U|_{X_{m}^{T}} \leq C\left|\left(\hat{u}_{0}, \hat{u}_{1}, \hat{g}\right)\right|_{Y_{m}^{T}}$. Since solutions of the wave equation in $K$ depend only on the data in the characteristic cone, then $\left(\widetilde{\square}^{\prime}\right)^{-1}\left(u_{0}, u_{1}, g\right)$ equals to the restriction of $U$ to $K$. This implies the assertions.

\footnotetext{
${ }^{2}$ This space is formed by restrictions to $K$ of functions from $C\left([0, T-a] ; H^{k}\left(B_{2 T}\right)\right)$.
} 
As above, we define a Banach space $Z_{m}^{\prime}$ as $\left(\tilde{\square}^{\prime}\right)^{-1} Y_{m}^{\prime}, Z_{m}^{\prime} \subset X_{m}^{\prime}$. Due to (2.1) $Z_{m}^{\prime}=$ $\operatorname{Tr}\left(Z_{m}^{T}\right)$. Denote by $\Phi^{\prime}$ the operator of the Cauchy problem (0.1), (0.2) on $K$ (cf. (1.5)). Then the following diagram is commutative

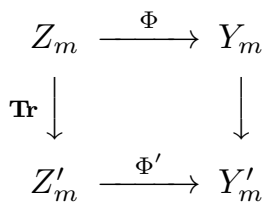

where the second vertical line stands for the mapping $\operatorname{Tr}_{T} \times \operatorname{Tr}_{T} \times \mathbf{T r}$. So we derive from Lemma 1.2 that for each $u \in Z_{m}$ the mapping $d \Phi^{\prime}(u)$ is an isomorphism. Hence, $\Phi^{\prime}$ is an analytic isomorphism

$$
\Phi^{\prime}: Z_{m}^{\prime} \gtrsim O
$$

where $O=\Phi^{\prime}\left(Z_{m}^{\prime}\right)$ is a domain in $Y_{m}^{\prime}$. We define $\mathcal{O}^{0}$ by the relation (1.9). This is a domain in $\mathcal{H}^{m}$ such that the problem (0.1), (0.2) has a solution $u \in X_{m}^{\prime}$ if and only if $\left(u_{0}, u_{1}\right) \in \mathcal{O}^{0}$. For $0 \leq \tau \leq T-a$ the mapping

$$
S_{0}^{\tau}\left(u_{0}, u_{1}\right) \mapsto(u(\tau), \dot{u}(\tau)), \quad \mathcal{O}^{0} \rightarrow \mathcal{H}^{m},
$$

is analytic since $\Phi^{\prime-1}$ is an analytic mapping on $\mathcal{O}$.

Similar we may consider eq. (0.1) on the smaller cone

$$
K^{\tau}=\left\{(t, x) \in[\tau, T-a] \times \mathbb{R}^{d}:|x| \leq T-t\right\}=K \cap\left([\tau, T-a] \times \mathbb{R}^{d}\right), \quad 0 \leq \tau<T-a .
$$

In this way we get a domain $\mathcal{O}^{\tau} \subset \mathcal{H}^{m}\left(K^{\tau}\right)$ such that eq. 0.10 has a solution $u(t, x),(t, x) \in K^{\tau}$, which is a trace on $K^{\tau}$ of a function from $X_{m}^{\prime}$, if and only if $(u(\tau), \dot{u}(\tau)) \in \mathcal{O}^{\tau}$. Clearly the flow-map $S_{0}^{\tau}$ is an analytic operator $S_{0}^{\tau}: \mathcal{O}^{0} \rightarrow \mathcal{O}^{\tau}$. In difference with Section 1 this is not an embedding.

Now we define families of the Cauchy problems and of the corresponding operators $\Phi^{\prime}$. Since the function $f$ analytically in $(t, x)$ extends to $U_{\varepsilon}(K)$, then eq. (0.1) analytically extends to a bigger cone

$$
K^{+}=\left\{(t, x) \in[-\varepsilon, T+\varepsilon-a] \times \mathbb{R}^{d}:|x| \leq T+\varepsilon-t\right\}, \quad \varepsilon>0 .
$$

For any $\theta \in B_{\varepsilon}=\{|\theta| \leq \varepsilon\} \subset \mathbb{R}^{d}$, as before, we denote by ${ }_{\theta} R$ the shift ${ }_{\theta} R(t, x)=(t, x+\theta)$, and set

$$
K^{\theta}={ }_{\theta} R(K) \subset K^{+}, \quad\left({ }_{\theta} R f\right)(t, x, u \nabla u, \dot{u})=f\left(t,{ }_{\theta} R x, u \nabla u, \dot{u}\right) .
$$

As before, ${ }_{\theta} \Phi^{\prime}$ is the operator of the Cauchy problem with the nonlinearity ${ }_{\theta} R f$. The mapping

$$
\bar{\Phi}^{1}: B_{\varepsilon} \times Z_{m}^{\prime} \rightarrow Y_{m}^{\prime}, \quad(\theta, u) \rightarrow{ }_{\theta} \Phi^{\prime}(u)
$$

is analytic. For each $\theta \in B_{\varepsilon}$ it defines an analytic diffeomorphism

$$
\bar{\Phi}^{1}(\theta, \cdot): Z_{m}^{\prime} \rightarrow{ }_{\theta} \mathcal{O} \subset Y_{m}^{\prime},
$$

which analytically depends on $\theta$, as well as its inverse.

Considering eq. (0.1) with the shifted nonlinearity ${ }_{\theta} R f$ in a smaller cone $K^{\tau}$ we define the corresponding domain ${ }_{\theta} \mathcal{O}^{\tau} \subset \mathcal{H}^{m}\left(b_{\tau}\right)$, formed by the initial data $(u(\tau), \dot{u}(\tau))$ for which the 
shifted equation has a solution in $K^{\tau}$, extendable to a function from $X_{m}^{\prime}$. Then the flow-map of the shifted equation ${ }_{\theta} S_{0}^{\tau}$ is an analytical mapping

$$
{ }_{\theta} S_{0}^{\tau}:{ }_{\theta} \mathcal{O}^{0} \rightarrow{ }_{\theta} \mathcal{O}^{\tau}
$$

which analytically depends on $\theta$, and ${ }_{\theta} S_{0}^{\tau} \circ{ }_{\theta} R={ }_{\theta} R \circ S_{0}^{\tau}$. That is, denoting by $u(t, x)$ a solution of (0.1), (0.2) we have

$$
u(t, x+\theta)=\left({ }_{\theta} S_{0}^{t}\right)\left(u_{0}(x+\theta), u_{1}(x+\theta)\right) .
$$

In particular, if the functions $u_{0}$ and $u_{1}$ are analytic in the closed ball $b_{0}$ (i.e. analytically extend to its vicinity in $\left.\mathbb{R}^{d}\right)$, then $u(t, x)$ is analytic in $x$.

By the Cauchy-Kowalewski theorem the solution $u(t, x)$ is analytic in the vicinity of the disc $b_{0} \times\{0\}$ in $\mathbb{R}^{d+1}$. Considering shifts of the nonlinearity $f$ by the time-translations and arguing as above (cf. Section 1.3) we find that $u$ is analytic in $t$. We have proven

Theorem 2.2. Assume that $f$ is an analytic function on $U_{\varepsilon}(K) \times \mathbb{R}^{d+2}$ for some $\varepsilon>0$, and that the Cauchy data $u_{0}, u_{1}$ are analytic in $b^{0}$. Let the Cauchy problem (0.1), (0.2) have a solution $u(t, x) \in X_{m}^{\prime}$. Then $u$ is analytic.

An obvious local version of Theorem 1.5 also is true.

Since the open non-truncated characteristic cone $K^{o}=\left\{(t, x) \in[0, T) \times \mathbb{R}^{d}:|x|<T-t\right\}$ is the union of the closed truncated cones $K\left(T^{\prime}, a\right)$ with $T^{\prime}<T$ and $a>0$, then a natural version of Theorem 2.2 holds for the cone $K^{o}$.

\subsection{Global problems}

In the assumptions of Theorems 1.7 let $R>0$ be such that any two points of the ball $B_{R}$ are not equivalent modulo the lattice $\Gamma$ (which defines the torus $\mathbb{T}^{d}$ ). Define $T^{\prime}=\min \{2 R, 2 T\}$ and cover the layer $\left[0, T^{\prime}\right] \times \mathbb{T}^{d}$ by finitely many truncated cones $K\left(2 T^{\prime}, T^{\prime}\right)$, shifted by vectors $(0, \xi), \xi \in \mathbb{R}^{d}$. Then by Theorem 2.2 the solution $u$ is analytic in the layer. Iterating this construction (if $T^{\prime}<T$ ) we see that $u$ is analytic in $[0, T] \times \mathbb{T}^{d}$. So the local results of this section provide another proof of Theorems 1.5 and 1.7 .

They also straightforwardly generalise to quasilinear wave equations in a connected open domain in an analytic Riemann homogeneous space, not necessarily compact. In this case $\square=\partial^{2} / \partial t^{2}-\Delta$, where $\Delta$ is the corresponding Laplace-Beltrami operator. Now the straight cone $K$ should be replace by the characteristic cone, constructed in terms of the geodesics of the corresponding metric, and the translations ${ }_{\theta} R$ - by the local isometies. This generalisation implies that Theorem 1.5 with $k=d$ and Theorem 1.7 remains true for quasilinear wave equations on a compact homogeneous analytic Riemann manifold $M$. For example, on the standard sphere $S^{d}$.

\section{Related results}

\subsection{Quasilinear parabolic equations}

The approach to study analyticity and partial analyticity of solutions in the space-variables, explained above, applies to other equations (to which the Cauchy-Kowalewski and Ovsiannikov- 
Nirenberg theorems do not apply). For example, to quasilinear parabolic equations

$$
\dot{u}-\Delta u+f(t, x, u, \nabla u)=0, \quad x \in \mathbb{T}^{d}, t \geq 0, \quad u_{t=0}=u_{0},
$$

where $f$ is sufficiently smooth in $t, x$ and is analytic in $u$ and $\nabla u$. As in Section 1.1] one can find suitable space $Z_{m}^{T}$ and $Y_{m}^{T}$ such that the operator $\Phi$ of the Cauchy problem (3.1) defines an analytic diffeomorphism between $Z_{m}^{T}$ and a subdomain of the space $Y_{m}^{T}$, see Kuk81, Kuk82. In the same way as in Section 2 we prove that if $f$ is analytical in the space-variables, then classical solutions of (3.1) with analytical initial data are space-analytic. This is a well known result, which holds true for $t>0$ without assuming analyticity of $u_{0}(x)$; we do not discuss the corresponding literature but only mention the papers Ang90b, Ang90a, where analyticity of solution for nonlinear parabolic equations is obtained, based on ideas, related to those in our work and in Kuk82. But we also can prove that if $f$ is analytic in $u, \nabla u$ and in a part of the space-variables, as well as the function $u_{0}$, then the solution $u(t, x)$ is analytic in these space variables. This result seems new. Note that the assertion of Theorem 1.7 does not hold for the problem (3.1), even when $f=0$, since a solution of the Cauchy problem (3.1) $\left.\right|_{f=0}$ with analytic $u_{0}(x)$ may be non-analytic in $t$ when $t=0$.

The approach applies to the Navier-Stokes system on the $d$-torus with $d=2$ or $d=3$, perturbed by a sufficiently smooth force $h(t, x)$, see Kuk82. It implies that if the initial data and the force $h$ are analytical in space-variables $x_{1}, \ldots, x_{k}$, where $1 \leq k \leq d$, then a corresponding strong solution $u(t, x)$ remains analytical in this space-variables till it exists. For $k=d$ this is well known, e.g. see in book [DG95].

Similar consider the $3 \mathrm{~d}$ NSE in the thin layer $M \times(0, \varepsilon)=\{(\varphi, r\})$, where $M=S^{2}$ or $M=\mathbb{T}^{2}$. At the boundary $(M \times\{0\}) \cup(M \times\{\varepsilon\})$ impose the Dirichlet or Navier boundary conditions. Let the force and initial data are

i) analytic in $\varphi$,

ii) bounded uniformly in $t \geq 0$, uniformly in $\varepsilon \in(0,1)$.

Due to Raugel-Sell (see TZ97 and references therein), if $\varepsilon>0$ is sufficiently small, then there exists a unique strong solution $u(t, \varphi, r), t \geq 0$. Our result implies that this solution is analytic in $\varphi$.

\subsection{NLS equations}

The result of Theorem 1.5 remains true for the nonlinear Schrödinger equation

$$
\dot{u}-i \Delta u+f(t, x, \operatorname{Re} u, \operatorname{Im} u)=0, \quad u_{t=0}=u_{0}, \quad x \in \mathbb{T}^{d},
$$

where the complex function $f$ is continuous in $t, H^{m}$-smooth in $x(m>d / 2)$ and real analytic in $\operatorname{Re} u, \operatorname{Im} u$. The proof of the theorem remains literally the same if we choose $X_{m}^{T}=C\left(0, T ; H^{m}\right)$, $Y_{m}^{T}=H^{m} \times C\left(0, T ; H^{m}\right)$ and proceed as in Section 1 (cf. Kuk81). As before, we can replace $\mathbb{T}^{d}$ by any homogeneous Riemann space, analytic and compact.

\subsection{Smooth and partially smooth solutions}

Results of Sections 1 and 3.1 concerning spatial analyticity and partial spacial analyticity of solutions remain true, with the same proof, for their spacial smoothness. For example if the 
nonlinearity $f$ and the initial data $u_{0}, u_{1}$ of the problem (0.1), (0.2) are smooth in the variables $x_{1}, \ldots, x_{k}, 1 \leq k \leq d$, and the problem has a solution $u(t, x) \in Y_{m}^{T}$, then $u$ also is smooth in $x_{1}, \ldots, x_{k}$. Similar, if for the Navier-Stokes system on $\mathbb{T}^{3}$ the initial data and the force are smooth in some variable $x_{l}$, then a corresponding strong solution is smooth in $x_{l}$ till it exists.

\section{Appendix: analytic maps between Banach spaces}

Notations for spaces and operators in this appendix are independent from the rest of the paper. Proofs of the two theorems, given below, may be found in [PT87], Appendix B.

Let $X$ and $Y$ be complex Banach specs, formed by certain classes of complex functions, and let $X^{R}$ and $Y^{R}$ be their subspaces, formed by real-valued functions (in the main part of the text we use a number of spaces like that). Let $O$ be a domain in $X$. As in the finite-dimensional case, a mapping $F: O \rightarrow Y$ is called (complex) analytic if each pint $x \in O$ has a neighbourhood $U \subset O$ such that the restriction of $F$ to $U$ may be written as a sum of series

$$
F(x+u)=\sum_{k=0}^{\infty} F_{k}^{(x)}(u), \quad x+u \in U
$$

Here $F_{k}^{(x)}: X \rightarrow Y$ is a bounded $k$-homogeneous mapping. That is, it equals to the restriction to the diagonal of some bounded and symmetric complex-linear mapping $X^{k}=X \times \cdots \times X \rightarrow Y$. Moreover, $\left|F_{k}^{(x)}(u)\right|_{Y} \leq C r^{k}|u|_{X}^{k}$ for suitable positive $r$ and $C$ (so the series above converges uniformly if $U$ is sufficiently small).

Definition of a real-analytic mapping $F: X^{R} \supset O^{R} \rightarrow Y^{R}$ is similar. It is easy to see that a mapping $O^{R} \rightarrow Y^{R}$ is real-analytic if and only if it extends to a complex-analytic map $O \rightarrow Y$, defined on some neighbourhood $O$ of $O^{R}$ in $X$. The theorem below gives a very convenient criterion of analyticity:

Theorem 4.1. A map $F: O \rightarrow Y$, where $O$ is a domain in $X$, is complex-analytic if and only if it is locally bounded ${ }^{3}$ and weakly analytic. The latter means that for each $x \in O, u \in X$ and any $\xi \in Y^{*}$ there exists $\varepsilon>0$ such that the function

$$
\{z \in \mathbb{C},|z|<\varepsilon\} \rightarrow \mathbb{C}, \quad z \mapsto \xi(f(x+\varepsilon u))
$$

is analytic.

Corollary 4.2. If $F$ is continuous, then it is sufficient to require analyticity of maps (4.1) only for a set of triplets $(x, u, \xi)$ which is dense in $O \times Y \times Y^{*}$.

Proof. For any given triplet $(x, u, \xi)$ as in Theorem 4.1 let us approximate it by a sequence of admissible triplets $\left\{(x, u, \xi)_{n}, n \geq 1\right\}$. Then the mapping (4.1) is a limit in the uniform topology of analytical mappings (4.1) $n$. So it is analytic.

Example 4.3. Let $X=H^{s}\left(\mathbb{T}^{d} ; \mathbb{C}\right), s>d / 2$, and let $f(x, u)$ be a continuous function on $\mathbb{T}^{d} \times Q$, where $Q$ is a neighbourhood of $\mathbb{R}$ in $\mathbb{C}$, analytic in $u, H^{s}$-smooth in $x$ and satisfying

i) $\|f(\cdot, u)\|_{s} \leq C(R)$ for each $u \in Q_{R}=\{z \in Q:|z|<R\}$ and for every $R>0$.

\footnotetext{
${ }^{3}$ That is, each point $x \in O$ has a neighbourhood, where $F$ is uniformly bounded.
} 
Let $O \subset X$ be the domain $O=O(R, \delta):=\left\{u \in X:\|u\|_{s}<R,\|\operatorname{Im}(u)\|_{s}<\delta\right\}, \delta<R$. Then the mapping $F: u(x) \mapsto f(x, u(x))$ is defined on $O$ if $\delta$ is sufficiently small in terms or $R$ (and $Q$ ). Since $s>d / 2$, then $X$ is a Banach algebra and $F: O \rightarrow Y$ is a continuous mapping. In particular $F$ is locally bounded. Moreover, if $O^{\prime}=O(R-\gamma, \delta-\gamma)$, where $\gamma<\delta$, then

$$
\|F(u)\|_{s} \leq K(s, d, R, C(R), \delta, \gamma) \quad \forall u \in O^{\prime} .
$$

For integer $s$ it is not hard to check this directly, for non-integer $s$ see e.g. RS96.

Let $u(x), v(x)$ and $\xi(x)$ be smooth complex functions on $\mathbb{T}^{d}$ such that $u \in O$. Consider the function

$$
z \mapsto \int_{\mathbb{T}^{d}} f(x, u(x)+z v(x)) \xi(x) d x,
$$

defined in the vicinity of $0 \in \mathbb{C}$. The integrand is bounded uniformly in $z, x$ and analytic in $z$. So the function above is analytic, and the mapping $F$ is analytic by Corollary 4.2 .

What was said implies that if i) holds and $f$ is real for real arguments, then this function defines a real-analytical mapping $F: X^{R} \rightarrow X^{R}$. Consider the space $Y=C(0, T ; X)$. For a similar reason $f$ defines a real-analytical mapping $Y^{R} \rightarrow Y^{R}, u(t, x) \mapsto f(x, u(t, x))$. Same is true if $f$ continuously depends on $t$.

Corollary 4.4. If $\mathrm{O}_{1}, \mathrm{O}_{2}$ are domains in complex Banach spaces $X_{1}, X_{2}$, then a mapping

$$
F: O_{1} \times O_{2} \rightarrow T
$$

is analytic if and only if it is locally bounded and for each $x_{1} \in O_{1}$ and $x_{2} \in O_{2}$ the mappings

$$
O_{1} \rightarrow Y, \quad x \mapsto F\left(x, x_{2}\right), \quad O_{2} \rightarrow Y, \quad x^{\prime} \mapsto F\left(x_{1}, x^{\prime}\right)
$$

are analytic.

Proof. In one direction the implication is obvious. Now let (4.3) be a locally bounded mapping such that the maps (4.4) are analytic. Take any $\left(x_{1}, x_{2}\right) \in O_{1} \times O_{2},\left(u_{1}, u_{2}\right) \in X_{1} \times X_{2}$ and $\xi \in Y^{*}$. Consider the function $\left(z_{1}, z_{2}\right) \mapsto \xi\left(F\left(x_{1}+z_{1} u_{1}, x_{2}+z_{2} u_{2}\right)\right)$, defined in a suitable bi-disc $\left\{\left|z_{1}\right|<\varepsilon_{1}\right\} \times\left\{\left|z_{2}\right|<\varepsilon_{2}\right\}$. It is analytic in $z_{1}$ for $z_{2}$ fixed, and vice versa. So this function is analytic by the Hartogs theorem, see Kra92. Accordingly $F$ is weakly analytic, so it is analytic.

For mappings in Banach spaces the analytic implicit function theorem remains true:

Theorem 4.5. Let a mapping (4.3) be analytic, and let for some $\left(x_{1}^{0}, x_{2}^{0}\right) \in O_{1} \times O_{2}$ its differential in second variable $d_{2} F\left(x_{1}^{0}, x_{2}^{0}\right): X_{2} \rightarrow Y$ be a linear isomorphism. Denote $F\left(x_{1}^{0}, x_{2}^{0}\right)=y^{0}$. Then in a suitable neighbourhood of $\left(x_{1}^{0}, x_{2}^{0}\right)$ in $O_{1} \times O_{2}$ the equation $F\left(x_{1}, x_{2}\right)=y^{0}$ defines $x_{2}$ as a unique analytic function of $x_{1}$.

\section{References}

[AM84] S. Alihnac and G. Metivier, Propagation de l'analyticité des solutions d'équations hyperboliques non-linéaires, Invent. Math. 75 (1984), 189-204. 
[Ang90a] S. Angenent, Nonlinear analytic semiflows, Proc. Roy. Soc. Edinburgh Sect. A 115 (1990), 91-107.

[Ang90b] _ Parabolic equations for curves on surfaces, Part I. Curves with p-integrable curvature, Ann. of Math. 132 (1990), 451-483.

[BB77] C. Bardos and S. Benachour, Domaine d'analyticite des solutions de l'équation d'Euler dans un ouvert de $R^{n}$, Ann. Scu. Norm. di Pisa (4) 4 (1977), 647-687.

[DG95] Ch. Doering and J. Gibbon, Applied Analysis of the Navier-Stokes equations, Cambridge University Press, Cambridge, 1995.

[Hör97] L. Hörmander, Lectures on Nonlinear Hyperbolic Differential Equations, SpringerVerlag, Berlin, 1997.

[Koc93] H. Koch, Mixed problems for fully nonlinear hyperbolic equations, Math. Z. 214 (1993), 9-42.

[Kow75] S. Kowalewski, Zur Theorie der partiellen Differentialgleichungen, J. Reine Angew. Math. 80 (1875), 1-32.

[Kra92] S. G. Krantz, Function Theory of Several Complex Variables, AMS Chelsea Publishing, Providence, Rhode Island, 1992.

[Kuk81] S. B. Kuksin, Diffeomorphisms of functional spaces that correspond to quasilinear equations, PhD Thesis, Moscow State University (1981).

[Kuk82] - Diffeomorphisms of functional spaces that correspond to quasilinear parabolic equations, Math. USSR Sbornik 117 (1982), 359-378.

[Nis77] T. Nishida, A note on a theorem of Nirenberg, J. Differential Geom. 12 (1977), 629633.

[PT87] J. Pöschel and E. Trubowitz, Inverse Spectral Theory, Academic Press, Boston, 1987.

[RS96] T. Runst and W. Sickel, Sobolev Spaces of Fractional Order, Nemytskij Operators, and Nonlinear Partial Differential Equations, Walter de Gruyter and Co, Berlin, 1996 .

[Sog08] C. Sogge, Lectures on Non-Linear Wave Equations, second ed., International Press, Boston, 2008.

[Tem97] R. Temam, Infinite-Dimensional Dynamical Systems in Mechanics and Physics, Springer-Verlag, Berlin, 1997.

[TZ97] R. Temam and M. Ziane, Navier-Stokes equations in thin spherical domains, Contemp. Math., AMS 209 (1997), 281-314. 